\title{
Learner and Teacher Roles in the Treatment of Oral Error in Group Work
}

\author{
Anthony Bruton \\ Institute of Education \\ Singapore \\ Virginia Samuda \\ University of Michigan \\ U.S.A.
}

It is now generally accepted that language learners' errors play a crucial role in both the learning process, by allowing the learner to test his hypotheses about the target language (TL), and in the teaching process, by supplying the teacher with information about what has or has not been mastered (Corder 1967). This approach to error does not accept the premise that all the learner's errors are due to interference only or that errors should be, where possible, either kept to a minimum or drilled out of existence.

However, the studies that have been made of 'approximative systems' (Nemser 1971), 'idiosynchratic dialects' (Corder 1967) and 'interlanguages' (Selinker 1972) suffer from major limitations when applied to the classroom. First, as Bell (1974) observes, their analysis of a single learner's interlanguage is both 'impossible and impracticable' because of its transient nature, and furthermore because the teacher is rarely responsible for only one learner. Second, they implicitly emphasize the learner's command of the forms of the language rather than adopt a broader perspective of the learner's ability to communicate in the $\mathrm{TL}$.

Although recent research has moved in a more positive vein towards a consideration of the treatment of error by teachers (Fanselow 1974, Cathcart and Olsen 1976, Allwright 1975), this too has been based on a number of inconsistencies. On the one hand, the behaviourist view of the effect of errors has been rejected, but on the other, their analyses are based on classrooms with a strong structuralist slant, definitely teacher-centred, with a focus on the forms of the language. The result is an emphasis on specific types of error and particular options open to the teacher at given 'crisis points' (Allwright 1975). There is an implicit assumption that the teacher is the only person to isolate errors and request treatments of them. This is the subject of the first part of this paper.

It we are to consider the effect of errors and the part they play in the learning process, we must look at the role of errors not just in terms of different language learning theories, but also, and as importantly, in terms of the methodology used to teach the TL. If a teacher adopts a strict structuralist methodology, it can be argued that his view of error and his treatment of it is largely predetermined, so that there will be an emphasis on drills and the elimination of errors when they become apparent. 
It is probably due to a consideration of this particular method that the elements isolated are errors of form at the expense of the broader problems of self-expression and miscommunication which arise when learners are involved in a communicative event. Furthermore, this method might preclude the types of treatment applied and the points at which they occur. By limiting research to this type of situation, the above-mentioned writers seem to be perpetuating a limited approach to language teaching, and also taking a restricted view of the skills and strategies a learner needs in order to communicate effectively in the TL. This is the subject of the second part of this paper.

As Richards (1971) points out, it is necessary to account for performance errors and errors in strategies of communication which 'derive from the fact that heavy communication demands may be made on the second language, forcing the learner to mould whatever he has of the second language into a means of saying what he wants to say or getting done what he wants to get done.' In a teacher-centred classroom where a structuralist me thodology is used, these 'potential' errors would not become apparent and therefore would not be remedied. This is especially so where the learner's command of the TL is limited, since such errors occur often only in more advanced classes where students are allowed to express themselves more freely.

It is clear then that attention could now more relevantly be turned to those learning environments where oral communication is an aim, where the teacher is not always the dominant participant and where the methodology provides contexts for learners to communicate meaningfully in the TL. In this paper we discuss some of the features arising in such situations, based on the observation of a group of adult learners from a range of $\mathrm{L1}$ backgrounds, attending an in-service English language course at the Institute for English Language Education, University of Lancaster. They were recorded on video over a period of one week while involved in a variety of problemsolving tasks, during which there was no teacher intervention.

In Section A we consider the errors that these learners treated themselves and the treatments they used; in Section B we consider the types of error they did not treat and discuss the implications of these findings for classroom methodology.

\section{Section A}

There was considerable evidence that the learners were capable of correcting each other successfully, even though they had not been instructed to do so by the teacher; furthermore, they employed a variety of different treatment strategies.

Before discussing the errors made and the treatment types employed, there are a number of points which need clarification. First, in referring to "error," we rely on our own native-speaker intuition as a rule of thumb. A non-native speaker perception would depend on the state of the development of his interlanguage and his concentration on the actual language being used around him. In the context under analysis, where communication rather than cor- 
rectness was the important factor, we would not have expected the learners to pay undue attention to the language, except when it hindered communication. An added complication for the language learner in this context is the fact that he has to pinpoint for himself or for his peers the errors made; this, of course, is done for him in the teacher-centred classroom.

This possibly accounts for the fact that a large number of errors passed unnoticed. However, for the purposes of this paper, we concentrate on the errors that were treated, in order to see what types of error they were and how they were treated. We further limited ourselves to treatment which occurred immediately or soon after the error was made, although there were instances of errors being gradually treated through peer influences over time, without it being possible to isolate their when, why or how. We have called this latter treatment type 'correction by permeation,' since the correct form gradually permeated a participant's idiolect during the task. For instance, one participant referred to a picture as a 'design' at the beginning of a task; he later adopted 'picture' after it had been used by peers in the meantime.

A peer treatment can be applied to either an incorrect form or to a correct one, in native-speaker terms. To a learner, however, both forms might appear incorrect. Interestingly, we only noted one case of a correct form being changed to an incorrect one through immediate treatment during the entire ten-hour period of observation.

In analyzing the data, our interest lay initially with the types of errors which were remedied, albeit in some cases, unsuccessfully, and the types of treatment the learners used.

\section{Error Types Corrected}

The errors corrected were classified in four broad categories; it is interesting to note that these categories are very close to the types normally treated by teachers in the classroom.

(a) Lexical

There were cases when a participant mis-selected a word or used a word from his $\mathrm{Ll}$ and a peer supplied an alternative. The most common occurrence was a pause or pause accompanied by a gesture, followed by an offer of a word by a peer. Although this might not be an error in the strictest sense, it nonetheless produced a treatment.

(b) Syntactic

These errors were mostly with tenses or verb forms where a correct form was offered and in a few cases, an alternative treatment. 
(c) Pronunciation

In these cases, a peer offered the correct form of a pronunciation error; in some cases, the error could also be categorized as syntactic or lexical, however. For instance, $/$ tok $/ \rightarrow /$ tuk/ or $/$ telk/?

(d) Understanding

These instances were when misunderstandings of the task instructions were immediately apparent as the participants tried to work out what they had to do in the task. Occasionally a participant did not understand a word/phrase a peer used, so clarification was sought.

\section{Error Treatment and the Outcome}

If we assume that an error has been made, then it is either treated or not. In the latter case, the outcome will be that the error remains. However, if it is treated, then there are a number of options open before the final outcome is reached. First, the student can correct himself; this could result in a further error, or alternatively, a correct result.

The second possibility is for a peer to offer a repair question with the expectation that a second attempt will be made. The error would stand if this attempt is not made; however, if it is, the outcome may be correct or incorrect. The third major possibility is where a peer offers a straight alternative. This offer may be correct or in correct. If the initial participant accepts it and it is correct, then he will have been successful. He might, however, not accept the peer's suggestion, in which case, his original error would stand.

Figure 1 should clarify any shortcomings in this explanation.

Figure 1: Error Treatment and Outcome.

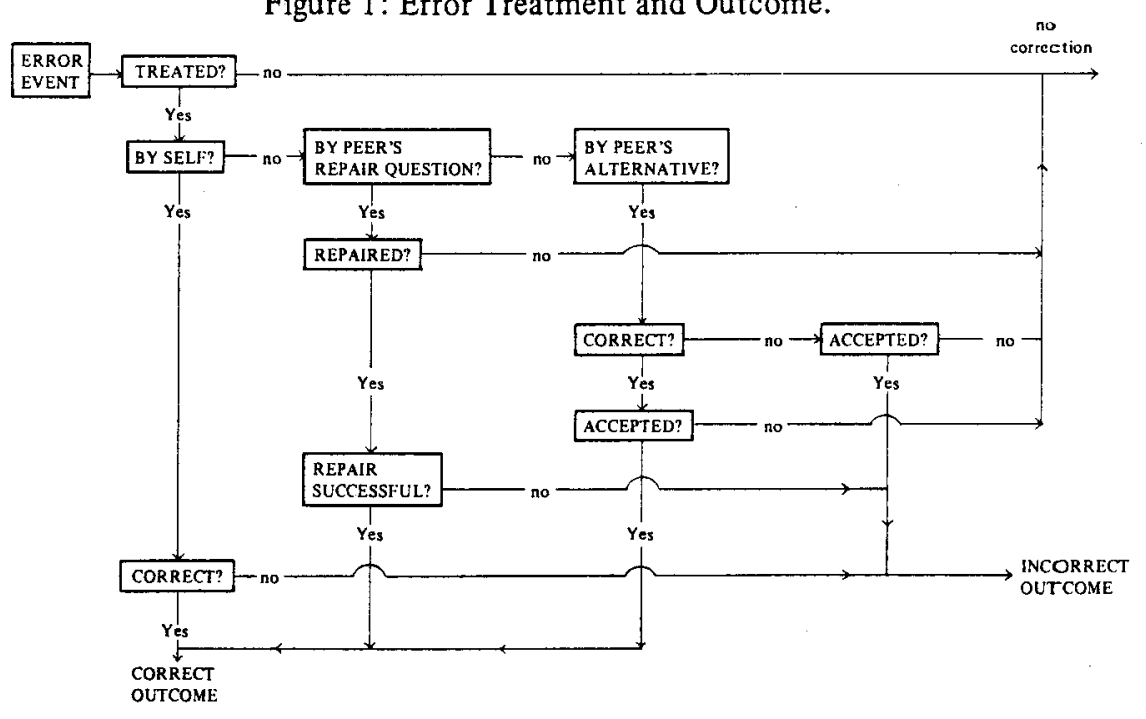




\section{The Different Types of Treatment}

In this section we have categorized the immediate treatment types under five headings, the first four being peer corrections. The categories are tentative but nevertheless show the range of treatments the students were able to use.

(1) Straight alternative by peer

In this type, a peer offered a direct correction to the student who made the error:
A: Well, he take the money, (he/tok/)
B: $\quad$ (he/trk/)
$\mathrm{C}$ : took the money, he took the money.

(2) Offered possibilities by peer

Here a peer offered a possible insertion, often in the pause, or pause plus gesture situation. It also occurred when an L1 word was used.
A: The chair have tree
B: Three
A: pieces of er
B: Stick?
A: Yeah.

(3) Repair question by peer

This is often in the form of a 'What?' or an 'I don't understand" question.
A: I cannot get more than two/r^loz/
B: only
A: Hmmm
C: Two what?
D: Two rules
B: (Two rules)
A: (Two rules) rules? (to D)
C: (rules)
D: (rules)
A: rules rA $1 ə z$.......huh huh (laughs at his original error)

(4) Rejection by peer

Here there was a rejection, followed by an alternative or explanation offered, or a reference to the text:

A: quickly he /distu:rb/

B: No, not 'disturb' you cann say

A: Ah, yes, blocked, blocked the the ehm 'blocked' 


\section{(5) Correction by self}

In this.case it was sometimes difficult to know if the original error was an error, or just a slip. There were also numerous instances of pauses followed by the student filling them himself, but these would not be necessarily subsumed under error. An example of what would be a self-correction, follows:

A: What about the leg of the /tsea/? Is it clear on the old man?

$\mathrm{B}$ : Are you following? (to $\mathrm{C}$ )

C: Which one?

B: (the)

A: (the) leg of the $/$ tsea/of the old man.

There are in fact numerous variations within the categories, but the examples should serve to show the versatility of the students' correction strategies, and the similarity with teacher correction procedures.

Some aspects of peer correction were of considerable interest. First the most frequent treatments were lexical items, mainly in the pause situation. To date, most research on the treatment of error has concentrated on syntactic or pronunciation errors, which in this sample did not occur very frequently. The pause seems to reflect a 'hiatus' (Varadi 1973) for the student as he discovers a gap in his interlanguage or is unable to retrieve a word on the spot. It reflects, furthermore, the importance of vocabulary in communication contexts where time is at a premium. Errors of understanding took much longer to remedy and seemed to cause the greatest problems. This is discussed in the following section. The number of treatments of pronunciation and syntactic errors were limited, but the fact that they occurred at all is significant.

A point which is relevant here for those concerned about the development of classroom pidgins was the fact that the students did not seem to pick up many errors from each other as most of the time they were not repeating what their peers said. This is true of most on-going communication and it was only in one task that there was considerable echoing of peer utterances, since they were checking each other's pictures in a "Spot the Difference" exercise.

\section{Section B}

In this section, we focus on those error types which the learners did not treat themselves. Our aim here is to consider the role of error in the communication process as a whole and in particular, the relationship between the notion of error as it has been traditionally defined and instances of actual communication breakdown between the participants we observed, thus leading us to reconsider what actually constitutes a "communicative error." 
A Model of Communication

Miller (1974) argues that for communication to take place, the individual has to draw on knowledge stored at six distinguishable levels. At the lower levels, this knowledge comprises the ability to hear acoustic signals and extract phonological, syntactic and lexical information about the language; at the higher levels, it includes conceptual knowledge and a system of beliefs against which to evaluate what is heard.

If this knowledge is organized on six levels, then there must be six distinguishable levels of linguistic processing at which communication can fail.

(1) Hearing : The individual may fail to respond to an auditory stimulus.

(2) Matching :The individual may be unable to match the utterance to a phonetic pattern.

(3) Accepting : The individual may be unable to accept the utterance as a grammatical sentence in the language.

(4) Interpreting : The individual may be unable to assign lexical meaning to the utterance or grasp what it entails or presupposes.

(5) Under- : The individual may be unable to understand the standing speaker's intentions or relate them to his conceptual understanding of the world.

(6) Believing : The individual may be unable to evaluate the utterance because his system of beliefs is not shared with the speaker.

It is clear, therefore, that levels (1)-(4) draw on what is linguistic knowledge, the domain of well-formed utterances, while levels (5)-(6) draw on pragmatic knowledge, the domain of well-formed ideas. Miller proposes that the most serious failures in communication between native-speakers are those which occur because of a failure to process at the higher levels.

It is when we apply this model to communication between FL learners that its implications become most interesting. The lower levels clearly constitute the very areas on which error analysts and teachers have traditionally drawn, seemingly at the expense of the higher levels, where, Miller argues, communication is most likely to fail.

Miller (1974) argues that communication is 'protected' at the lower levels because linguistic knowledge, contextual information and redundancy all contribute to provide the hearer with in-built error-correcting devices, which work as an 'antidote' against communication failure in 'correcting' the signal he receives. At the higher levels, however, redundancy is introduced 
in more abstract forms, for instance through the use of intentional verbs to underline illocutionary force and through recourse to logic.

It seemed relevant to apply this model to learner discourse for several reasons. First, since our concern was with the crisis points in communication breakdown, we felt it was more relevant to draw on a psychological rather than a purely linguistic model. Second, the mere classification of error into discrete units of syntax, lexis and phonology is clearly an inadequate means of measuring their effect on the communicative whole. Miller's emphasis on the dynamic interaction between levels of processing, on the other hand, might be able to characterize the cumulative effect of a discrete unit of error on the whole. The application of such a model, then, should place emphasis on the process rather than the product of communication failure.

The pedagogical implications involved here now begin to emerge more clearly; such an emphasis might help the teacher isolate the roots of a communication failure and provide him with clues as to how to develop learners' redundancy strategies as an 'antidote' at the higher levels, for it certainly seems possible that results drawn from the knowledge stored at the higher levels may be used, in fact, to facilitate processing at the lower levels. This should lead us to reconsider the concept of 'communication error' and the teacher's role in the treatment of such error.

\section{Hierarchies of Error}

A model such as the one established by Miller underlines the need to establish some sort of hierarchy in the consideration and subsequent treatment of error, wherein attention is paid primarily to those errors which affect communication. There is nothing new in this notion, of course; Burt and Kiparsky's well-known distinction between global and local errors (1972) is an obvious example. However, we believe that attempts to establish such hierarchies, while claiming to lay emphasis on communicative goals, have to date, rarely moved beyond what constitutes in Miller's terms the the lowest levels of linguistic processing. Consequently, the crisis points in communication breakdowns are largely ignored. Burt and Kiparsky's classification of errors, for instance, operates in such a way that it is impossible to deal effectively with extended stretches of discourse and, in consequence, with whole communicative acts and with the ways in which these link together. As noted in Section A, such bases for analysis presuppose a classroom methodology rooted in sequences of teacher elicitation, response and feedback. This is an insufficient basis of analysis for the type of classroom we have described. While it is obvious that the teacher will need to establish a hierarchy in the errors he wishes to treat, we believe such a hierarchy must take in to account not only the types of error that do not disrupt communication (because they are 'protected' by context or redundancy), but also those errors which learners are capable of treating themselves. (See Section A.) This should form part of an overall teaching strategy in which learners gradually take responsibility for the treatment of these 'minor' errors, leaving the teacher free to concentrate on the more complex types of error. 
The complexity of these more complex types of error is the subject of the following sub-sections.

\section{Process and Product in Communication Breakdown}

We argue, then, that despite a declared interest in communication approaches to error such as the one outlined above, the focus still has been on the product and not on the processes which cause communication to fail. A product-centred approach to error tends to reflect a methodology in which learners are trained to jump through hoops at Miller's levels (1)-(4), producing well-formed sentences often at the expense of well-expressed ideas. As long as such an approach to error persists in giving precedence to the discre te units of an error, it will be unable to help teachers and learners towards a control of the communicative whole. We are interested, therefore, in evaluating the feasibility of a process-centred approach to error. For these reasons, our starting point was not with instances of error made, but with instances of communication breakdown.

\section{Types of Communication Breakdown} sample:

We noted four distinct types of communication breakdown in the

(i) apparent: The breakdown was immediately apparent to the (immediate) participants; repairs were employed and communication was resumed. (See Section A.)

(ii) apparent: The breakdown took longer to repair since it (delayed) took longer for the participants to perceive that communication had failed.

(iii) apparent: Although the participants were aware that some(partial) thing was going wrong, they were unable to pinpoint the cause correctly. In the attempt to resume the flow of communication they misrepaired. This generally had the effect of compounding the misunderstanding; eventually it became impossible to continue.

(iv) non-apparent: This type of breakdown was covert and participants remained unaware that they were miscom. municating. The most common instances of this type were linked with mismatching, when an individual mismatched a phonemic pattern and selected an alternative which closely approximated the original pattern.

If this alternative was then processed at all levels successfully subsequent communication was based on a different set of presuppositions. For instance, one participant mismatched "leader" as "reader". 
(The participants are discussing group roles for the completion of a task.)

B: OK. No. I mean do you want to be the leader?

C: You are the leader

D: No, what for? I - you mean I'm going to have to read this one?

B: Yes.

B's final response here is based on the presupposition that reading might well be one of the roles of the leader in that task. However, during the task the other participants often voiced frustration at $\mathrm{D}$ not assuming the role of leader. $\mathrm{D}$, on the other hand, was satisfied that he had fulfilled the role of reader successfully and could not understand this frustration. This is a clear example of communication failing at a lower level (in Miller's terms), and being compounded at a higher level.

\section{Communication Breakdown: An Example}

The most interesting, and most complex, type of breakdown was type (iii): apparent (partial). This is also the most problematic type of breakdown for both teachers and students to "treat."

To illustrate more precisely why this should be so, a small section of a communication breakdown of this type is included. It is impossible to discuss the entire breakdown here since it spanned seven minutes of discourse. Instead, we will deal with a short extract which has been taken from a fifteenminute problem-solving task, in which the participants (a group of four) were trying to agree on an interpretation of the events in a sequence of photographs. We do not intend to analyze this in depth, but we will consider the process of misunderstanding in the extract and focus on three key points in this process: See Figure 2, lines 1-5; lines 13-25 and lines 36-42.

The transcription has been laid out to make it possible to trace the development of this process. We have isolated on the left-hand side of the page those statements which relate directly to the development of the story/ interpretation the participants are working on. To them, this was the focal point of the task. On the right, we isolate the repairs used by the participants in the effort to maintain communication. The arrows indicate the complex process of reference and mis-reference that went on.

At a superficial level, the most extractable item in this transcript is the discussion on lines 13-25 of the meaning of eyesight, since the participants' interpretation of it is clearly "wrong." However, examination of the data clearly shows that the meaning of eyesight is not the issue; merely a stage in a long process of misunderstanding. 
Figure 2: Process of Misunderstanding.

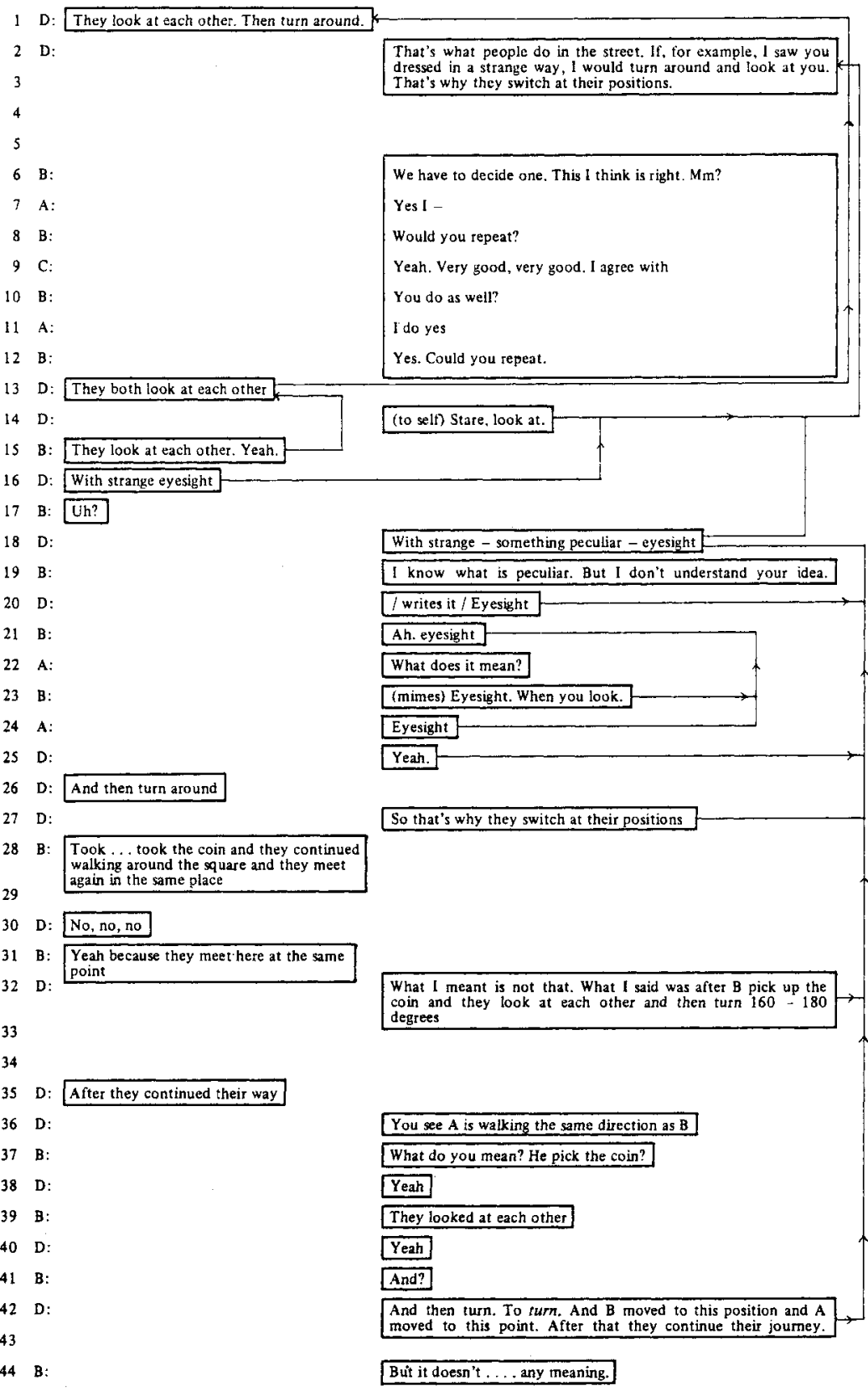


This starts on lines $2-5$, where $D$ clarifies the point made in line 1. The introduction of eyesight at line 15 is clearly a lexical error; but it is much more than this since D never directly refers back to his explanation in lines 2-4. In his attempts to elucidate his idiosyncratic interpretation of eyesight, he compounds the misunderstanding further. At line 25, his 'yeah' appears to be in agreement to his view of eyesight; it is taken by the others as approval of theirs. This becomes clearer (to the analyst but evidently not to the participants) at line 32 , where, using a repair strategy, D tries to 'correct' their interpretation by rephrasing his explanation. However, the focus of this explanation is misplaced. He tries to clarify the meaning of turn as lucidly as possible, but does not relate it to his concept of eyesight, thus making it difficult for the hearers to draw the parts together. His repair strategy fails because he does not appear to have a clear enough sense of the discourse as a whole for it to actually repair anything. In lines $36-42$, it does seem, momentarily, as if understanding might be restored by B's questioning of each part of D's interpretation. However, this only elucidated what had already been established between them: it is clear, at line 42 , that the idea cannot develop further because in these attempts at clarification, D once more omits reference to why they 'turned,' possibly because he felt he had explained it adequately in line 2 and because the others had professed to understand it. B's reaction at line 45 is, in this context, highly understandable.

Several important points emerge here. First, it is likely that most teachers would isolate and treat the obvious lexical errors here: eyesight and turn. However, in doing this, they would be adopting a product rather than a process centred approach to the whole. They would be attempting to treat communication at Miller's lowest levels of processing without taking into account that these errors are in fact compounding misunderstanding at the higher levels and isolating these will not help learners to come to terms with the whole.

More interestingly, we should note the repair strategies used by the participants; lines 18-19 encapsulate the basic issue here.

D: with strange - something peculiar eyesight.

B: I know what is peculiar. But I don't understand your idea.

In the face of misunderstanding, D's strategy is to elucidate at the level of discrete item - item by item. (This strategy is noted again in his attempt to clarify meaning at line 42D: And then turn, [pause] To turn.)

In response to B's request for clarification of the idea, he simply proceeds to the next item - eyesight - and makes no attempt to show the relationship between the items strange and eyesight. He assumes, further, that because B clearly understands the discrete meanings of strange and eyesight, then she must necessarily understand the whole message.

This highlights an interesting area of cause in communication breakdown. It is possible that the type of repair strategies employed here in fact reflect the types of treatment employed by teachers in the classroom. The 
teacher who treats errors as discrete items, focusing on product at the expense of process, may influence the way learners repair in contexts where the teacher does not play the dominant role.

A teacher-type repair, which concentrates on Miller's lowest levels is all that is available to learners as a model for the treatment of communication breakdown or miscommunication occurring at the higher levels. There is evidence to support this in the examples of peer correction types in Section $A$, where at times learner treatments closely parallel teacher treatments in type. In Section A, these treatments were often successful because they occurred at points where communication was not endangered. However, these error treatments are inadequate when employed as repair strategies and this inadequacy, in fact, serves to generate further misunderstanding.

\section{Implications for the Classroom}

How then does this affect the communicative classroom? First, by a reconsideration of the implications of a 'communication error.' The most serious type of error and the most serious cause of communication breakdown was what could be termed a cumulative error, that is to say, one whose disruptive effect was cumulative over a span of discourse. We noted that in isolation these errors appeared insignificant (that is, eyesight). But if viewed in isolation they would have been accorded insignificant treatment by the teacher and the relationship to the communicative whole would have been lost. Therefore, although the moment or fact of error might be insignificant in itself in formal terms, its effect is important in cumulative terms. It is thus impossible to treat such errors in any of the ways normally discussed in the literature (Allwright 1975, Cathcart and Olsen 1976).

Miller argues that to make communication work, the individual has to have access to a full range of error-detecting and error-correcting redundancies. These then should form part of the content of any course with communication as a goal. While it is obvious that the learner whose TL exposure is limited to teacher-centred classrooms is illequipped to deal with communication breakdown, it is also clear that even learners in communicatively oriented classroom settings will not, by that fact alone, be any better equipped to deal with such breakdowns either. From the outset learners should be made aware of the fact that communication breakdown is a normal feature of face-to-face interaction and they must be taught to both expect it and deal with it when it occurs. The inclusion of this in the teaching syllabus becomes especially important when we consider the practical difficulties involved in a teacher attempting to monitor and supply effective post-performance feedback on extended tasks as we describe them. Some areas to be covered might include:

(i) explaining: rephrasing of the message, focusing on overall meaning rather than discrete points within it.

(ii) checking: that the intended message has been received in terms of overall meaning rather than discrete problematical items. 
(iii) clarifying: seeking and giving clarification of the whole rather than the parts.

This then becomes part of a two-step teaching plan. First, learners work with materials which specifically highlight the types of strategy they will have to develop in communication. This is followed by the learners working on their own in groups on tasks so the teacher can check their overall performance and afterwards provide feedback. The teacher should also consider the range and type of task the learners are asked to perform. While problem-solving tasks will most obviously give rise to meaningful communication between learners, the lexical and structural demands of the tasks set must be taken into account. We found that tasks which had a common referent (for example, a picture) led to fewer communication breakdowns, as one would expect given Miller's model of communication. This then could provide a rational basis for the sequencing of material over a period of time. If a higher level of abstraction is gradually introduced in to the tasks, then heavier demands will be slowly made on the learners' repair strategies and in this way, the teacher is also maintaining some measure of indirect control over their output.

Finally, the importance of post-performance feedback cannot be overemphasized. The nature of this feedback will be largely determined by the priority the teacher accords errors to be treated. If he accords highest priority to those errors which disrupt communication, he cannot treat them as discrete items of error at the phonological, syntactic or lexical levels. He must instead evaluate the cumulative effect they have across a stretch of discourse. In order to make this task a manageable one, he should first consider what can be done most usefully and by what treatments; he also needs to consider by whom. He should take into account, therefore, the learners themselves as potential sources of treatment, both as an ongoing factor during groupwork and through an outside peer observer. In the case of the former, the learners would initially need some direction from the teacher, since as we noted, peer-correction tends to be haphazard when left to its own devices. In the case of using a peer observer, one way of doing this is to appoint an observer for each group to record instances of errors made. This is particularly effective if the observer has been briefed to record occurrences of a specific type of error rather than error 'in general.' In putting both of these strategies into action, the teacher is giving himself the freedom to monitor the learners' overall communication strategies and to check on the areas which would still require attention.

We have argued, then, that the notion of error in non-teacher centred classrooms needs to be fully reconsidered, not only in terms of methodology and treatment but also in terms of the roles adopted by learners and teachers as treaters of errors. Learners can take responsibility for the treatment of 'minor' product-centred errors but this must also be balanced by the teacher's focus on 'major' process-centred errors. Unless overall methodology and the teacher's total treatment strategies are fully considered, it will be impossible to indicate to learners the full significance of the errors they make in relation to the process of communication. 


\section{References}

Allright, R.L. 1975. Problems in the study of teacher's treatment of learner error. New directions in second language learning, teaching, and bilingual education: On TESOL 75, ed. by M. Burt and H. Dulay, 96-109. Washington, D.C.: TESOL.

Bell, R.T. 1974. Error analysis: a recent pseudoprocedure in applied linguistics. ITL: Review of Applied Linguistics $25: 26$. 35-49.

Burt, M and Kiparsky, C. 1972. The Gooficon: a repair manual for English. Rowley, Mass.: Newbury House.

Cathcart, R.L. and Olsen, J.E.W.B. 1976. Teachers and students' preferences for correction of classroom conversation errors. On TESOL 76, ed. by J. Fanselow and R. Crymes, $41-53$. Washington, D.C.: TESOL.

Corder, S.P. 1967. The, significance of learners' errors. IRAL (International Review of Applied Linguistics in Language Teaching) 5:4. 161-170.

Fanselow, J.F. 1974. The treatment of error in oral work. Paper presented at the eighth annual TESOL Convention, Denver.

Miller, G.A. 1974. Psychology, language and levels of communication. Human communication: theoretical explorations, ed. by A. Silverstein, 1-17. New York: J. Wiley.

Nemser, W. 1971. Approximative systems of foreign language learners. IRAL (International Review of Applied Linguistics in Language Teaching) 9:2.115-123.

Richards, J.C. 1971. Error analysis and second language strategies. Language Sciences 1\%. 12-22.

Selinker, L. 1972. Interlanguage. IRAL (International Review of Applied Linguistics in Language Teaching) 10:3. 209-232.

Varadi, T. 1973. Strategies of target language learner communication; message adjustment. Paper at the sixth conference of the Rumanian English Linguistics Project, Timisoura, Rumania. 